\title{
Radio Observation of Venus at Meter Wavelengths using the GMRT
}

\author{
Nithin Mohan ${ }^{\mathrm{a}}$, Subhashis Roy ${ }^{\mathrm{b}}$, Govind Swarup ${ }^{\mathrm{b}}$, Divya Oberoi ${ }^{\mathrm{b}}$, Niruj Mohan Ramanujam ${ }^{\mathrm{b}}$, Suresh Raju C.,*, Anil \\ Bhardwaj $^{\mathrm{a}}$
}

${ }^{a}$ Space Physics Laboratory, Vikram Sarabhai Space Center, Thiruvananthapuram-695022, India.

${ }^{b}$ National Center for Radio Astrophysics, Tata Institute of Fundamental Research, Pune-411007, India.

\begin{abstract}
The Venusian surface has been studied by measuring radar reflections and thermal radio emission over a wide spectral region of several centimeters to meter wavelengths from the Earth-based as well as orbiter platforms. The radiometric observations, in the decimeter $(\mathrm{dcm})$ wavelength regime showed a decreasing trend in the observed brightness temperature $\left(T_{\mathrm{b}}\right)$ with increasing wavelength. The thermal emission models available at present have not been able to explain the radiometric observations at longer wavelength $(\mathrm{dcm})$ to a satisfactory level. This paper reports the first interferometric imaging observations of Venus below $620 \mathrm{MHz}$. They were carried out at 606, 332.9 and $239.9 \mathrm{MHz}$ using the Giant Meterwave Radio Telescope (GMRT). The $T_{\mathrm{b}}$ values derived at the respective frequencies are $526 \mathrm{~K}, 409 \mathrm{~K}$ and $<426 \mathrm{~K}$, with errors of $\sim 7 \%$ which are generally consistent with the reported $T_{\mathrm{b}}$ values at $608 \mathrm{MHz}$ and $430 \mathrm{MHz}$ by previous investigators, but are much lower than those derived from high-frequency observations at 1.38-22.46 GHz using the VLA.
\end{abstract}

Keywords: Venus, surface, Radio Observations, Radiative transfer

\section{INTRODUCTION}

Venus being the nearest planet, its dense atmosphere and the surface have been the subject of many studies, including the ones from orbiting spacecraft, landers and from Earth-based observations in the last six decades:

(i) orbiting spacecraft, Mariner (Fjeldbo et al., 1971); Magellan (Pettengill et al., 1991); Venus Express (Markiewicz et al., 2007)

(ii) Venera and Vegas Landers (Vinogradov et al., 1976; Florenskii et al., 1982; Basilevsky et al., 1986), and

(iii) radar and radio observations made from the Earth (Campbell et al., 1989; Condon et al., 1973; Muhleman et al., 1973; Butler et al., 2001).

The atmosphere of Venus is comprised of $\sim 96 \% \mathrm{CO}_{2}$, $\sim 4 \%$ of $\mathrm{N}_{2}$ and trace amounts of gases like $\mathrm{H}_{2} \mathrm{O}, \mathrm{SO}_{2}$, $\mathrm{CO}$ and $\mathrm{H}_{2} \mathrm{SO}_{4}$ (Seiff et al. 1980; Butler et al. 2001). The thick atmosphere generates a pressure of $\sim 90$ bars at the surface. Since $\mathrm{CO}_{2}$ is a very efficient greenhouse gas, the surface is extremely hot, $\sim 735 \mathrm{~K}$; and for the same reason, it does not have a significant diurnal as well as equator to pole variation of temperature. Many studies have reported possible characteristics of the interior of Venus and its tectonic nature, as well as the possible areas of the Venus surface that expel its internal heat (Sinclair et al., 1970; Marov, 1978; Seiff et al., 1980; Phillips and Malin, 1983). It is postulated that heat generation from its core and by radioactive elements is similar to that for the Earth or Mars. Surkov (1983) has summarized studies of Venus

\footnotetext{
*Corresponding author

Email address: c_sureshraju@vssc.gov . in (Suresh Raju C.)
}

rocks by Venera 8, 9 and 10 that landed on its surface during 1972-75. The mean contents were found to be close to that of the basalts and granites of the Earth's crust having a density of $2.8 \pm 0.1 \mathrm{~g} \mathrm{~cm}^{-3}$.

The Venusian surface has been explored since 1961 with the first radar observation of Venus from Earth carried out at NASA's Goldstone Observatory (Carpenter, 1964). The successive radar observations revealed important information about Venus, such as its rotation is retrograde and the rotation period is 243.1 days, its axis of rotation is almost perpendicular to its orbital plane, and the planetary radius is $\sim 6,052 \mathrm{~km}$ (Goldstein and Carpenter, 1963).

Besides the ground-based radar observations, Venus surface has been mapped using spacecraft-based radars on the Pioneer 10 (Pettengill et al., 1980; Muhleman et al., 1979; Ford and Pettengill, 1983); and the Magellan probe (Pettengill et al., 1991; Campbell, 1994). All these studies were limited to single frequency and in horizontal $(\mathrm{H})$ polarization. Studies of radar echoes at longer wavelengths ranging from $15 \mathrm{~cm}$ to $7.84 \mathrm{~m}$ showed global mean surface reflectivity values of $\sim 0.15$. The low reflectivity of $\sim 0.02$ at $3.8 \mathrm{~cm}$ was attributed to the attenuation of radar echoes by the atmosphere at these higher frequencies (Pettengill et al., 1980). These radar measurements also indicated the presence of a thin layer (of $\sim$ centimeter thickness) of porous powdered soil or dust (Pettengill et al., 1988). The Magellan radar (SAR) data have been used extensively to characterize the Venusian surface by studying geomorphology and variation in the dielectric properties of the high and lowland regions. The dielectric permittivity at lowlands are $\sim 5$ showing the presence of dry basaltic or granite minerals, but high value of dielectric permittivity $(>50)$ at highlands indicates the presence of highly conducting mineral deposits or the presence of 
less absorbing materials that can return most of the incident radar signals back at these locations. These radar investigations showed that about $15 \%$ of the impinging radiation is reflected, indicating that the dielectric permittivity of Venus is in the range of $\sim 4.15$ to $\sim 4.5$ (Pettengill et al., 1992).

Several investigations have been made to derive properties of the thermal radio emission of Venus using Earth-based and space-borne radio telescopes operating at centimeter and decimeter wavelengths (Kuzmin, 1983; Condon et al., 1973; Muhleman et al., 1979; Pettengill et al., 1992; Butler et al., 2001). The passive mode operation of the Magellan radar enabled the measurement of radio emissivity at $12.6 \mathrm{~cm}$ wavelength in horizontal polarization for more than $91 \%$ of the Venus surface during the first 8 months of its operation (Pettengill et al., 1992). The global mean value of emissivity observed using horizontal linear polarization is 0.845 , a value that corresponds to a dielectric permittivity of between 4.0 and 4.5 , depending on the surface roughness. These values are consistent with the permittivity values of dry basaltic minerals that compose the bulk of the Venus surface. The above emissivity value is also in good agreement with that derived from the radar reflectivity.

Kuzmin (1983) summarized early radiometric measurements of the brightness temperatures $\left(T_{\mathrm{b}}\right)$ of Venus made using Earth-based radio telescopes. Butler et al. (2001) measured Venusian $T_{\mathrm{b}}$ at different frequencies by using the VLA observations at 22.46, 14.94, 8.44, 4.86 and $1.385 \mathrm{GHz}$ with errors of $\sim 2$ to $5 \%$. At shorter wavelengths $(<5 \mathrm{~cm}$ or frequencies above $6 \mathrm{GHz})$ radiation arises primarily from the dense atmosphere of Venus but at longer wavelengths (decimeter and meter wavelengths) thermal radiation is increasingly dominated by the surface and subsurface emission (Warnock and Dickel, 1972). Butler et al. (2001) proposed a detailed model, to explain the VLA measured values of $T_{\mathrm{b}}$ considering absorption at microwave frequencies by the atmosphere based on the vertical profiles of $\mathrm{SO}_{2}$ and $\mathrm{H}_{2} \mathrm{SO}_{4}$ derived from the Pioneer-Venus (PV) probes and that inferred from measurements of the Mariner V (MV) spacecraft (Muhleman et al., 1979). At longer wavelengths, the contribution of radiation from the surface and subsurface of Venus is also considered. However, their model predicts $T_{\mathrm{b}}$ values much higher than those measured at $1.385 \mathrm{GHz}$ and at $<1 \mathrm{GHz}$ by others. Muhleman et al. (1973), and Condon et al. (1973) measured $T_{\mathrm{b}}$ values at frequencies $<1 \mathrm{GHz}$ and found them to be in the range $500-550 \mathrm{~K}$. These $T_{\mathrm{b}}$ values are significantly lower than those measured at higher frequencies. The radiometric measurements made during 1972 - 73 used Wyllie's flux density scale for calibrations and doubts were raised in the literature about the scale used those measurements at frequencies < $1 \mathrm{GHz}$ (Condon et al., 1973). However, subsequently Baars et al. (1977) noted that "Wyllie's flux density scale is only $3 \%$ above our CasA scale". It is to be noted that Baars et al. (1977) flux density scale is widely used today by radio astronomers across the world for flux density calibration.

The depth of penetration of micro/radiowaves into the Venusian regolith depends on the dielectric properties of the same. The lander based in-situ measurements, Earthbased radar/radiometric measurements as well as the orbiter measurements concluded that the Venusian surface is dry and has low dielectric constant values of $\sim 4.5$. With this consideration the observation at meter wavelengths are suitable for the study of deeper subsurface characteristics since the penetration depth, $\delta$ (in meters), of the radiation, is related to the wavelength by the equation

$$
\delta=\frac{\lambda_{0} \sqrt{\varepsilon^{\prime}}}{2 \pi \varepsilon^{\prime \prime}}
$$

where $\varepsilon^{\prime}$ is the real part of the and $\varepsilon^{\prime \prime}$ is the imaginary part of the dielectric and $\lambda_{0}$ is the wavelength in free space. It has been found that there is a significant decrease in $T_{\mathrm{b}}$ beyond a wavelength of $\sim 15 \mathrm{~cm}$. There were a large number of successful observations carried out between several millimeters $(\mathrm{mm})$ to centimeter $(\mathrm{cm})$ wavelength. But no observations were reported beyond $70 \mathrm{~cm}(\lesssim 400 \mathrm{MHz})$ due to an increased system and background noise, solar interference and weak planetary emission (Condon et al., 1973).

Flux density measurements based on interferometric imaging do not suffer from base-level variations, solar interference and local radio-frequency interference which often afflict single dish observations (Condon et al., 1973), the latter two can also afflict the non-imaging interferometric observations (Muhleman et al., 1973). Here we report on interferometric observations carried out at 3 different wavelengths $50 \mathrm{~cm}, 90 \mathrm{~cm}$ and $123 \mathrm{~cm}$ (or $606 \mathrm{MHz}$, 332.9 MHz and 239.9 MHz, respectively) using Giant Meterwave Radio Telescope (GMRT). The $T_{\mathrm{b}}$ of Venus measured from images made from these observations can serve as inputs for developing an improved thermal emission model that can account for the increasing subsurface thermal emission at longer wavelengths. These are the first reported interferometric imaging flux density and $T_{\mathrm{b}}$ of Venus at frequencies lower than $620 \mathrm{MHz}$.

This paper presents the results obtained from analyzing the archival data of Venus (project code 05BBA01) collected during the observations made in March 2004 using the GMRT at $239.9 \mathrm{MHz}, 332.9 \mathrm{MHz}$ and $606 \mathrm{MHz}$, as discussed in Sections 2 and data processing and reduction are presented in Section 3. The results are presented in Section 4 , followed by discussion and conclusions in Sections 5 and 6 , respectively.

\section{OBSERVATIONS}

Observations of Venus were carried out on six days between March 20 and March 27, 2004 using the GMRT at three frequencies centered close to $239.9 \mathrm{MHz}$, $332.9 \mathrm{MHz}$ and $606 \mathrm{MHz}$. The $239.9 \mathrm{MHz}$ and $606 \mathrm{MHz}$ the observations were conducted simultaneously using the dual frequency co-axial GMRT feeds. The design of the GMRT is described in Swarup et al. (1991). Briefly, GMRT consists of 30 fully steerable parabolic dishes each of $45 \mathrm{~m}$ in diameter, 14 of which are located in a central 
array of $\sim 1 \mathrm{~km} \times 1 \mathrm{~km}$ extent and the other 16 in a Yshaped array of extent $25 \mathrm{~km}$. Two of the six days of observations had short observing runs, only the observations from the other four days are presented here. An integration time of 16.8 second and a spectral resolution of 125 $\mathrm{kHz}$ was used for these observations. Table 1 summarizes the observation details.

Both 3C147 and 3C48 were used as primary flux calibrators at 606.0, 606.1 and 239.9 MHz and only 3C48 was used for this purpose at $332.9 \mathrm{MHz}$. The primary flux calibrators were observed at the start and end of each observing session. The flux density of the flux calibrators was obtained from the task SETJY in the Astronomical Image Processing System (AIPS) and Common Astronomy Software Applications (CASA), which use Scaife and Heald flux density scale (Scaife and Heald, 2012) below $500 \mathrm{MHz}$ and Perley and Butler scale (Perley and Butler, 2013) above $500 \mathrm{MHz}$. These scales are in close agreement with Baars et al. (1977) scale at frequencies $\sim>300 \mathrm{MHz}$. The compact radio source $0318+164$ was used as both the phase and bandpass calibrator, it was observed for $\sim 5$ mins every 30 minutes.

Rather than tracking Venus, whose right ascension (RA) and declination (Dec) change with time, the antennas tracked the RA, Dec corresponding to the coordinates of Venus at the middle of observing period for that particular day. The ephemeris details of the Venus during the observations are tabulated in Table 2. The diameter of Venus varied from $21.19 \operatorname{arcsec}$ to $22.97 \operatorname{arcsec}$ over the 6 days of observations.

\section{DATA REDUCTION}

\subsection{Analysis Methodology}

To build confidence in our analyses and results, we use both CASA and AIPS and somewhat different analysis procedures. The observations made at 606.1 and 239.9 MHz were analyzed using both AIPS and CASA, those at $332.9 \mathrm{MHz}$ were analyzed only using AIPS and those at $606.0 \mathrm{MHz}$ were analyzed only using CASA. Data editing was performed to remove records highly deviant in amplitude, arising from man-made radio interference, in both time and frequency domains. The flagging of data analyzed in CASA was carried out using a combination of automated flagging outside CASA using FLAGging and CALibration (FLAGCAL), a software pipeline developed to automate the flagging and calibration of GMRT (Prasad and Chengalur, 2012; Chengalur, 2013), and manual flagging in CASA. The data analyzed in AIPS were first manually flagged for dead antennas followed by the automated flagging task RFLAG. First, we describe the analysis procedure followed in CASA. As mentioned earlier, we tracked the mean RA, Dec of Venus for every observing day. While this allowed Venus to move appreciably with respect to the phase center and in the antenna beam over the course of the observations, tracking at the sidereal rate enables us to do self-calibration to correct for the phase changes introduced by the GMRT electronics and the ionosphere during the observations. The background celestial radio sources seen in the field of view were used for selfcalibration. Next, CASA task UVSUB was used to subtract contribution of the background sources from the selfcalibrated visibility data using the statistically significant deconvolved (CLEAN) components in the model. An often used technique for imaging a source with non-sidereal motion (Venus) is to make multiple individual images, each of them over a duration short enough that the angular displacement of the source in that period is significantly smaller than the resolution of the imaging instrument. This method was followed for the 606.0, 606.1 MHz and 239.9 MHz observations. Synthesized beams at 606.0 and $606.1 \mathrm{MHz}$ were about 6 arcsec and about $15 \operatorname{arcsec}$ at 239.9 MHz. The angular velocity of Venus in RA and Dec was about 2.65 arcsec/minute and individual images were made every one minute. After primary beam correction, all the one-minute maps were aligned using the position of Venus available from the NASA JPL Horizons ephemeris (http://ssd.jpl.nasa.gov/horizons.cgi) and coadded. Finally, the co-added map of Venus was deconvolved using a point-spread-function corresponding to unflagged data for the entire duration of the observation.

The analysis strategy used in AIPS is common with that followed in CASA till self-calibration and subsequent removal of the contribution of background sidereal sources using UVSUB. The motion of Venus across the beam is accounted for by using a different strategy. An artificial strong point-source $(10 \mathrm{Jy})$ was added to the last spectral channel of the dataset. The position of this artificial source was shifted every minute in a direction equal and opposite to that of movement of Venus in the sky plane. Then, using a script, phase-only self-calibration was carried out only on the last spectral channel of this dataset using this artificial point source and a solution interval of one minute. The antenna solutions determined arise entirely due to the motion of the artificial source and were applied to all the frequency channels of the UV dataset. This changed the visibility phases of the UVSUB-ed data precisely by the amount needed to compensate for the motion of Venus. The last spectral channel, where the artificial source was introduced, was not used for imaging of Venus. Both of these analyses at $606.1 \mathrm{MHz}$ on $26 \mathrm{March}$, 2004 , using independent software suits and differing procedures, yielded flux densities which differ by $\leq 5 \%$ with similar images.

\subsection{Flux calibration uncertainties}

Given the challenging nature of these observations, particular attention was paid to estimating the uncertainties in flux calibration. In general, the errors in flux density of Venus as measured by an interferometer comprising physically large antennas like the GMRT arise from the following main reasons: (i) instrumental 'gain' variations due to the change in pointing directions between the primary and secondary calibrator, and also between the secondary calibrator and Venus; (ii) gain variations over time; and (iii) uncertainty in absolute flux density scales used to estimate the flux density of the primary calibrator. Each of these concerns is discussed briefly below. To quantify the net observed variation of the antenna gains during 
Table 1: GMRT observations of Venus 2004

\begin{tabular}{ccccc}
\hline $\begin{array}{c}\text { Central } \\
\begin{array}{c}\text { Frequency } \\
(\mathrm{MHz})\end{array}\end{array}$ & Date & $\begin{array}{c}\text { Bandwidth } \\
(\mathrm{MHz})\end{array}$ & $\begin{array}{c}\text { No. of } \\
\text { working } \\
\text { antennas }\end{array}$ & $\begin{array}{c}\text { Observing } \\
\text { time on } \\
\text { Venus (mins) }\end{array}$ \\
\hline 606.1 & March 26 & 16 & 26 & 311 \\
606.0 & March 27 & 16 & 26 & 334 \\
332.9 & March 19 & 16 & 28 & 210 \\
332.9 & March 20 & 16 & 27 & 240 \\
239.9 & March 26 & 8 & 26 & 311 \\
\hline
\end{tabular}

Table 2: Ephemeris data for GMRT observations of Venus 2004

\begin{tabular}{cccccc}
\hline $\begin{array}{c}\text { Central } \\
\begin{array}{c}\text { Frequency } \\
(\mathrm{MHz})\end{array}\end{array}$ & Date & $\begin{array}{c}\text { Venus Angular } \\
\text { Diameter }(\theta) \\
(\operatorname{arcsec})\end{array}$ & $\begin{array}{c}\text { Right Ascension } \\
\text { (mid-observation) } \\
\text { (h:min:s) }\end{array}$ & $\begin{array}{c}\text { Declination } \\
\text { (mid-observation) } \\
\text { (deg.arcmin.arcsec) }\end{array}$ & illumination \\
\hline 606.1 & March 26 & 22.72 & $3: 14: 48$ & +21.05 .00 & 52.9 \\
606.0 & March 27 & 22.97 & $3: 18: 59$ & +21.25 .33 & 52.3 \\
332.9 & March 20 & 21.40 & $2: 50: 35$ & +18.57 .51 & 55.9 \\
332.9 & March 19 & 21.19 & $2: 46: 30$ & +18.35 .06 & 56.4 \\
239.9 & March 26 & 22.72 & $3: 14: 48$ & +21.05 .00 & 52.9 \\
\hline
\end{tabular}

these observations, we measured the gains of the antennas from uncalibrated data towards the secondary calibrator observed on $19 \mathrm{March}, 2004$ at $332 \mathrm{MHz}$. Over the 5 hours of observations of the secondary calibrator, a gain variation of $\sim 10 \%$ was found. Typically for large dishes, the gain changes with elevation angle and these gain variations are correlated across different antennas. For the purpose of estimating uncertainties in flux density measurement, we assume these variations to be $100 \%$ correlated. Given that the flux density is directly proportional to the square of the gain, the resulting uncertainty in the flux density estimates cannot be larger than $\sim 20 \%$. However, observing the primary and secondary calibrators at similar elevations and frequent observations of the secondary calibrator reduce this uncertainty substantially as discussed later in this section.

We make the conservative assumption of the gain change being linear with elevation angle. During the observations, the elevation of the secondary calibrator changed from $\sim 85^{\circ}$ to $\sim 23^{\circ}$ (a change of $\sim 62^{\circ}$ ). The primary calibrator was observed at the start of observation at an elevation angle of $67^{\circ}$. Attributing the entire observed gain variation of $\sim 10 \%$ over the elevation range covered to elevation angle dependence of GMRT dishes, the difference between the elevation angles of the two calibrators implies a gain change of $\sim 2.5 \%$. As the angular distance between Venus and the secondary calibrator was much smaller $\left(\sim 8^{\circ}\right)$, a similar argument leads to an expected elevation angle dependent gain variation between these two sources to be $\sim 1 \%$.

In addition, the instrumental gains might also drift over time. The gains vary slowly and smoothly in time and we assume this variation to be proportional to the difference in time over time scales of interest here. In order to track these gain variations, the observations of primary and secondary calibrators were done within 30 minutes of each other and the secondary calibrator was observed every 30 minutes. Assuming all of the $10 \%$ of the gain variation to come from such gain drifts in time, the estimated change in gain due to the time difference while observing primary and secondary calibrator, and secondary calibrator and Venus is $<1 \%$.

The absolute flux density scale is now believed to be accurate to better than 3\% (Scaife and Heald, 2012). Since all the above-mentioned errors add in quadrature, we finally get an error in measured flux density of Venus to be $\sim 7 \%$. We note that in practice the elevation dependent gain variation is shallower than linear dependence assumed here. It varies much more slowly near the zenith, where the absolute gain from the primary was used to calibrate the flux density of the secondary calibrator. In addition, the gain variations in time of each antenna are independent and hence expected to contribute randomly to the uncertainty in the measured flux density. Both of the above-mentioned factors will reduce the uncertainty in flux density estimate when compared to the estimate presented.

\subsection{Uncertainties in Galactic background temperature}

The full sky map at $408 \mathrm{MHz}$ made by Haslam et al. (1983) was used to estimate the Galactic background temperature, $T_{\text {gal }}$, towards the direction of Venus. The $408 \mathrm{MHz} T_{\text {gal }}$ towards Venus was $30 \mathrm{~K}$ during our observations. Considering the uncertainty in the zero level and absolute calibration, the uncertainty on Haslam et al. (1983) measurement is $\sim 4 \mathrm{~K}$. The spectral index of $T_{\text {gal }}$ near the location of Venus is measured to be $-2.6 \pm 0.15$ (Reich and Reich, 1988). This leads to a $T_{\text {gal }}$ of $10 \pm 1.5$, $52 \pm 7$ and $122 \pm 22 \mathrm{~K}$ at $606,332.9$ and $239.9 \mathrm{MHz}$, respectively.

The position of Venus itself in the sky changed by $\sim 5-10^{\prime}$ during observations and the $T_{\text {gal }}$ can vary as a function of the Galactic latitude and longitude $(1, b)$. The Haslam et al. (1983) map has a resolution of $0.85^{\circ}$ and any variation of $T_{\text {gal }}$ at smaller angular scales is averaged out in this map. However, $T_{\text {gal }}$ variations at angular scales of 
$1-30^{\prime}$ are easily picked up by GMRT $330 \mathrm{MHz}$ band observations. To estimate this variation, we subtracted out the background extragalactic sources at high resolution and then made a low-resolution map of the region at 332 MHz. The resultant map had a resolution of $159^{\prime \prime} \times 135^{\prime \prime}$. The rms of the map was $\sim 3 \mathrm{mJy} /$ beam, which corresponds to a fluctuation of $<2 \mathrm{~K}$ in $T_{\text {gal }}$ at $330 \mathrm{MHz}$ at $\sim 3-30^{\prime}$ scales. No structures at scales $>5^{\prime}$ were seen with a significance $>2 \sigma$. Therefore, the uncertainty due to angular variations in $T_{\text {gal }}$ scaled to the above frequencies is low in comparison to the base level uncertainty in Haslam et al. (1983) map.

\subsection{Contamination from background sources}

Most background sources tend to have spectral indices which will make them brighter at lower frequencies, where we find Venus to be weaker. So we have carefully examined the effects of removal of these sources. We note a few things to build confidence that our results are not significantly affected by the errors due to background subtraction:

1. We find that the background sources are all unresolved sources, which greatly simplifies the deconvolution problem.

2. The $r m s$ in the UVSUB maps including regions from where sources have been subtracted are similar to the $r m s$ in the final map of the Venus. This implies that any residual flux left behind after cleaning is small enough to not give rise to any discernible artifacts even at the lowest frequency.

3. There are few background sources close to Venus. Hence, any contamination from them can only be due to the side lobes of the point-spread-function (PSF).

4. When using CASA to make the final maps for the Venus, we cut out the appropriate parts of the 1 minute maps and align them to ensure that flux from Venus falls in the same pixels. This has the consequence that any small residual flux from the background sources will get smeared in the Venus maps, further reducing any contamination from them. Similarly, when using AIPS, though the implementation details differ, the methodology followed ensures the residual flux from the background sources will get smeared over a region spanning the track of the Venus in the sky plane.

\section{RESULTS}

Figure 1 shows the map of Venus at $606.1 \mathrm{MHz}$ from the observations of March 26, 2004 and Figure 2 shows the map of Venus at $332.9 \mathrm{MHz}$ from the observations of 19 March 2004. The error on the measured flux density of Venus was obtained by multiplying the measured rms noise in the background image by $\sqrt{N}$, where $N$ is the total surface area of Venus measured in units of the synthesized beam. We then multiply by a factor of 1.07 to take care of the random and systematic errors as discussed earlier. From the known solid angle subtended by Venus during these observations (Table 2), its brightness temperature, $T_{\mathrm{b}}$, can be estimated from the observed values of its flux density using Rayleigh-Jeans law.

However, an additive correction needs to be applied to the $T_{\mathrm{b}}$ thus determined. To understand its origin, we note that the $u v$ coverage of any interferometer has a central hole, reflecting the absence of baselines shorter than some minimum length. This leads to the common situation that the peak of the point-spread-function (PSF) is surrounded by a shallow negative bowl, or equivalently the interferometer is not sensitive to brightness distribution at large angular scales. A practical consequence of this is that the interferometer resolves out the smooth Galactic background and when the PSF is convolved with an extended source like Venus, the source is observed to be sitting in a bowl of negative flux (Taylor et al., 1999). Also, the Galactic background radiation gets fully absorbed by Venus (Condon et al., 1973). Together, they lead to an underestimate in the true value of the $T_{\mathrm{b}}$ of Venus by an amount equal to the temperature of the Galactic background, $T_{\text {gal }}$, which is resolved out by the interferometer and needs to be added to get the true brightness temperature for Venus, $T_{\mathrm{b} \text {,cor }}$. We used the $T_{\text {gal }}$ values as discussed in Sec. 3.3.

The $T_{\mathrm{b} \text {,cor }}$ values for the three GMRT frequencies are provided in Table 3. Column 1 lists the frequency of observation, column 2 the date of observation and columns 3 and 4 are the size and the position angle of the synthesized beam, respectively. Column 5 gives the measured rms in the map of Venus, Columns 6 and 7 are measured flux density and the estimated rms error of the flux density in the map, respectively. Column 8 gives the $T_{\mathrm{b}}$ computed from the measured flux density and the size of the known sources, the magnitude of the correction for $T_{\text {gal }}$ is given in column 9. Column 10 gives the final computed values of the brightness temperature of Venus, $T_{\mathrm{b} \text {,cor }}$. In column 11, the numbers in bold give the average value of $T_{\mathrm{b}}$ for a given frequency. As discussed in Sec. 3.2, 7\% error is assumed to account for random and other systematic errors. The lowest frequency data $(239.9 \mathrm{MHz})$ was analyzed using both CASA and AIPS which gave similar rms in the image plane. In both the cases, Venus could not be detected in the image. A $3 \sigma$ value is used to place an upper limit of the brightness temperature of Venus at this frequency and is indicated by $\downarrow$ in Table 3 .

Figure 3 and Table 4 compile all available measurements of brightness temperature of Venus in the wavelength range from $0.013 \mathrm{~m}(22.46 \mathrm{GHz})$ to $1.25 \mathrm{~m}(239.3$ $\mathrm{MHz}$ ), including the ones obtained by us. Figure 3 also includes the model by Butler et al. (2001). Our observations clearly indicate that $T_{\mathrm{b} \text {,cor }}$ decreases with increasing wavelength beyond $\sim 0.5 \mathrm{~m}$, in contrast to the model which remains practically flat beyond $\sim 0.06 \mathrm{~m}$. 
Table 3: Summary of $T_{\mathrm{b}}$ measurements (2004)

\begin{tabular}{|c|c|c|c|c|c|c|c|c|c|c|}
\hline $\begin{array}{c}\text { Frequency } \\
(\mathrm{MHz})\end{array}$ & Date & $\begin{array}{c}\text { Beam Size } \\
(\operatorname{arcsec})\end{array}$ & $\begin{array}{c}\text { Beam } \\
\text { Position } \\
\text { Angle }\left(^{\circ}\right)\end{array}$ & $\begin{array}{c}\text { Map } \\
r m s \\
(\mathrm{mJy})\end{array}$ & $\begin{array}{l}\text { Flux } \\
(\mathrm{mJy})\end{array}$ & $\begin{array}{c}\Delta \text { Flux } \\
(\mathrm{mJy})\end{array}$ & $T_{\mathrm{b}}(\mathrm{K})$ & $T_{\mathrm{gal}}(\mathrm{K})$ & $T_{\mathrm{b}, \text { cor }}(\mathrm{K})$ & $\begin{array}{c}\text { mean } \\
T_{\mathrm{b}, \text { cor }} \\
(\mathrm{K})\end{array}$ \\
\hline 606.1 & March 26 & $6.5^{\prime \prime} \times 5.1^{\prime \prime}$ & 53.7 & 0.45 & 58.0 & 1.68 & 540 & $10 \pm 1.5$ & 550 & \\
\hline 606.0 & March 27 & $5.5^{\prime \prime} \times 4.2^{\prime \prime}$ & 57.0 & 0.56 & 54.0 & 2.54 & 492 & $10 \pm 1.5$ & 502 & $526 \pm 22$ \\
\hline 332.9 & March 19 & $13.8^{\prime \prime} \times 11.0^{\prime \prime}$ & 87.2 & 0.40 & 10.6 & 0.65 & 376 & $52 \pm 7$ & 428 & \\
\hline 332.9 & March 20 & $13.5^{\prime \prime} \times 11.4^{\prime \prime}$ & 81.6 & 0.50 & 9.7 & 0.82 & 337 & $52 \pm 7$ & 389 & $409 \pm 33$ \\
\hline 239.9 & March 26 & $15.4^{\prime \prime} \times 13.3^{\prime \prime}$ & 89.0 & 1.50 & $\downarrow 4.74$ & 1.50 & $\downarrow 282$ & $122 \pm 22$ & $\downarrow 426$ & \\
\hline
\end{tabular}

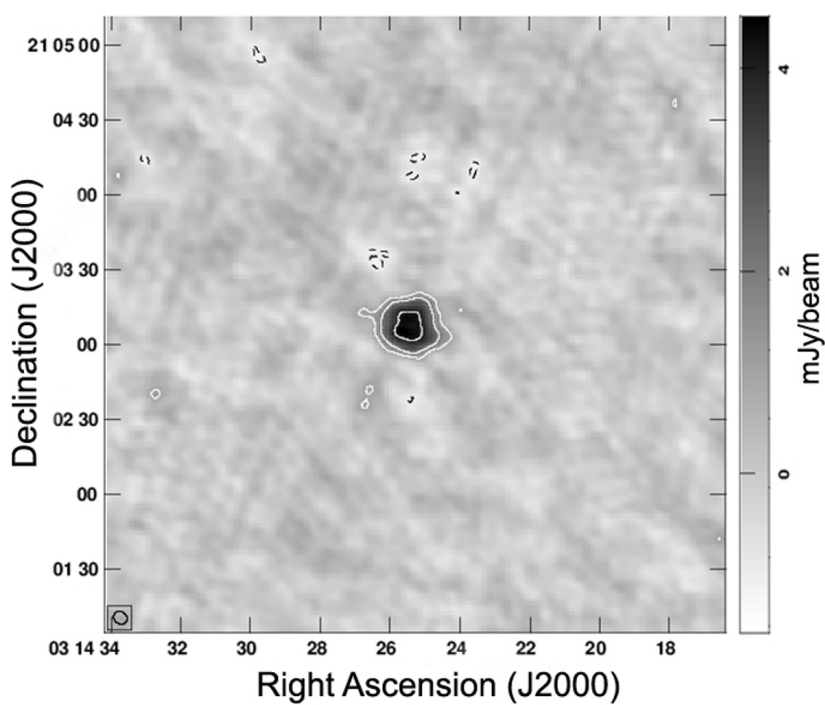

Figure 1: Image of Venus made at $606.1 \mathrm{MHz}$ on 26 March 2004 using the GMRT. Contour values are -2, -1, 1, 2, 4, 6 mJy. Peak flux value is $5.93 \mathrm{mJy}$ and the total flux density is $58.0 \mathrm{mJy}$. The rms value of the image $=0.45 \mathrm{mJy}$.

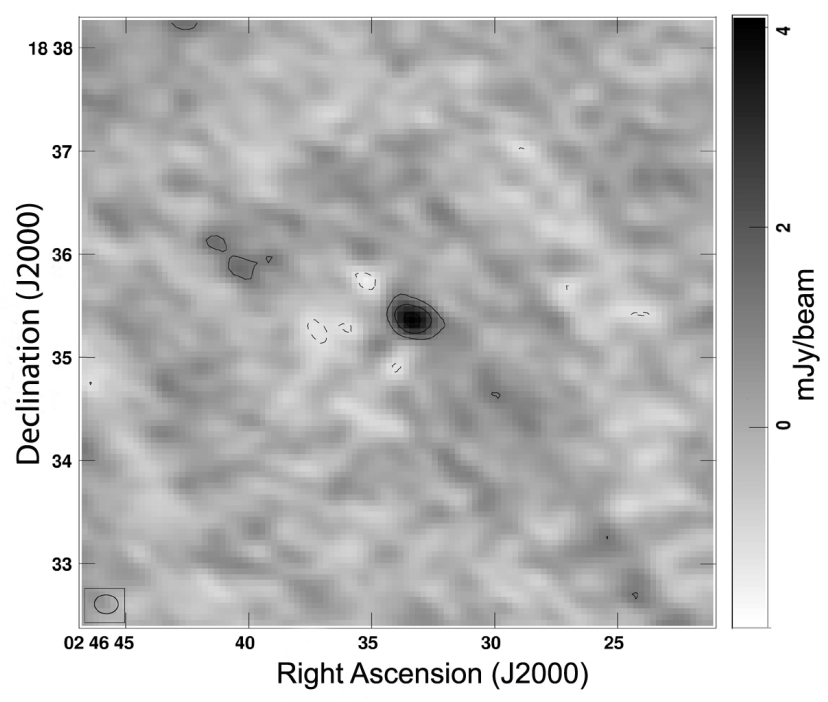

Figure 2: Image of Venus made at $332.9 \mathrm{MHz}$ on 19 March 2004 using GMRT. Contour values are -2,-1,2,3,4,6 mJy. Peak flux value is $4.07 \mathrm{mJy}$ and the total flux density is $10.6 \mathrm{mJy}$. The $r m s$ value of the image $=$ $0.4 \mathrm{mJy}$.

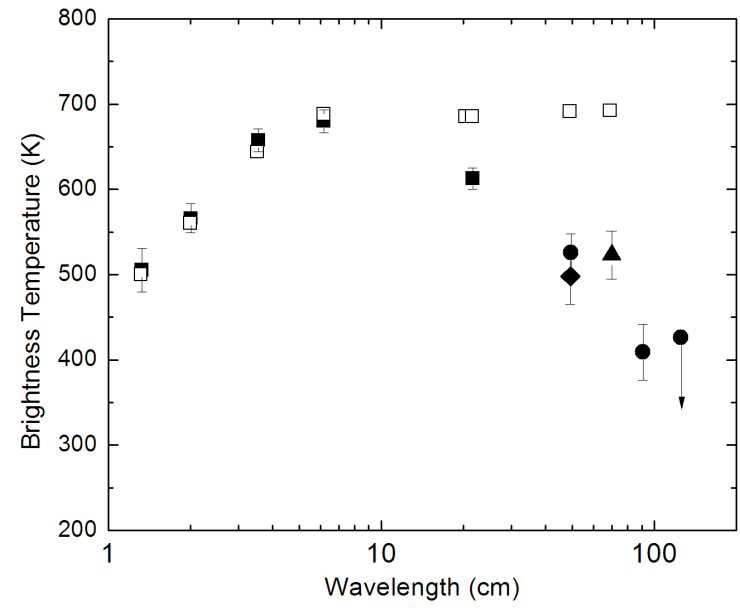

Figure 3: Plot of derived brightness temperature, $T_{\mathrm{b}}$, versus wavelength. (घ) shows measured values and $(\square)$ shows the model calculations by Butler et al. (2001) using the VLA. The $T_{\mathrm{b}}$ value as obtained by Muhleman et al. (1973) is shown in ( $\bullet$ ), that by Condon et al. (1973) is marked by $(\boldsymbol{\Delta})$ and (๑) shows values obtained using the GMRT (this work). Vertical lines show \pm one $r m s$ values.

\section{DISCUSSION}

As can be seen from Table 4 and Figure 3 that the $T_{\mathrm{b}}$ of Venus obtained from GMRT observations at longer wavelengths and those reported values by earlier investigators (Condon et al., 1973; Muhleman et al., 1973), are appreciably lower than those observed by Butler et al. (2001) at $\mathrm{cm}$ wavelengths (at frequencies $>1.385 \mathrm{GHz}$ ). The detailed model $T_{\mathrm{b}}$ values as seen in Figure 3 agree very well with the observed values by Butler et al. (2001) at shorter wavelengths $(<6 \mathrm{~cm})$ increasing with the same in a loglinear manner with a slope of $\sim 40 \mathrm{~K} / \mathrm{cm}$ which peaks around $6 \mathrm{~cm}$. The observations beyond $11 \mathrm{~cm}$ wavelength again show a decreasing $T_{\mathrm{b}}$ values in a log-linear form. But the model by Butler et al. (2001) predicts the values of $T_{\mathrm{b}}$ at lower frequencies (or longer wavelengths) to be same as that at $\sim 6 \mathrm{~cm}$.

Very low atmospheric opacity, nearly $0.006 \pm 0.005$ at $608 \mathrm{MHz}$ and about 0.02 at $1.4 \mathrm{GHz}$ were reported by Muhleman et al. (1973) and Butler et al. (2001), respectively. As the GMRT observations are at and around these frequencies, it can be safely assumed that the atmosphere 
Table 4: Derived values of $T_{\mathrm{b}}$ as a function of frequency and wavelength

\begin{tabular}{ccccc}
\hline $\begin{array}{c}\text { Frequency } \\
(\mathrm{MHz})\end{array}$ & Wavelength $(\mathrm{m})$ & $T_{\mathrm{b}}(\mathrm{K})$ & Investigators & Radio Telescopes \\
\hline 22460 & 0.013 & $505.2 \pm 25.3$ & Butler et al. (2001) & VLA \\
14940 & 0.020 & $565.9 \pm 17.0$ & Butler et al. (2001) & VLA \\
8440 & 0.036 & $657.5 \pm 13.2$ & Butler et al. (2001) & VLA \\
4860 & 0.062 & $679.9 \pm 13.6$ & Butler et al. (2001) & VLA \\
1465 & 0.204 & $619.0 \pm 12.4$ & Butler et al. (2001) & VLA \\
1385 & 0.217 & $612.8 \pm 12.3$ & Butler et al. (2001) & VLA \\
606 & 0.495 & $526 \pm 22$ & This work & GMRT \\
608 & 0.493 & $498 \pm 33$ & Muhleman et al. (1973) & Owen's valley \\
430 & 0.698 & $523 \pm 28$ & Condon et al. (1973) & Arecibo 300m dish \\
332.9 & 0.909 & $409 \pm 33$ & This work & GMRT \\
239.9 & 1.250 & $<426$ & This work & GMRT \\
\hline
\end{tabular}

is almost transparent at the GMRT frequencies. Another possible mechanism responsible for the reduction in $T_{\mathrm{b}}$ at radiowaves was the presence of selective wavelength absorbing ionosphere as suggested by Kuzmin $(1964,1967)$. However, this suggestion was ruled out by Warnock and Dickel (1972) based on the Mariner V electron density profile measurements (Herman et al., 1971) which showed a low peak electron density of $5.2 \times 10^{5} \mathrm{~cm}^{-3}$ at an altitude of $135-140 \mathrm{~km}$, which was not enough to act as an absorber. Muhleman et al. (1973) and Condon et al. (1973) in their independent investigations reported the insignificance of the Venusian ionosphere at lower frequencies at $\sim 608 \mathrm{MHz}$ and $430 \mathrm{MHz}(70 \mathrm{~cm})$ so that the Venusian atmosphere including the ionosphere can be neglected in determining the $T_{\mathrm{b}}$ at low frequencies.

The other possible reasons for the reduction in $T_{\mathrm{b}}$ with wavelength observed in the radio observations at decimeter wavelength could be the variations in the dielectric constant or temperature. The radar signal can be significantly affected by the reflection and absorption depending on the dielectric properties of the surface medium, whereas the scattering is controlled by the surface roughness. It must be noted that the temperature plays only a minor role in the variation of the radar signal. Based on radar observations at $50 \mathrm{MHz}$ and $38 \mathrm{MHz}$ (Klemperer and Bowles, 1964; James and Ingalls, 1967; James et al., 1967), Condon et al. (1973) have ruled out a drastic variation in the values of the dielectric constant of the Venusian regolith at least up to several tens of meters. The dielectric constant measurements of typical planetary rocks including basalts at $450 \mathrm{MHz}$ and $35 \mathrm{GHz}$ by Campbell and Ulrichs (1969) revealed no significant variation in the dielectric properties with frequency. They have also ascertained the absence of absorbing lines which can alter the dielectric values in between these two frequencies.

Warnock and Dickel (1972) using their two-layer subsurface model tried to explain the reduced radar reflectivity at centimeter wavelength and reduced brightness temperature at decimeter wavelength. The best fit in explaining the reduced reflectivity at $\mathrm{cm}$ wavelength and $T_{\mathrm{b}}$ at decimeter wavelength was obtained when a two-layer model consisting of a layer with $\varepsilon=1.5$ overlaying another layer of $\varepsilon=8.31$ was chosen. However, a better fit to the observation for $\lambda>15 \mathrm{~cm}$ was obtained when a possible decreasing radiating temperature with increasing subsurface depth was assumed. This is expected as at longer wavelengths the emission is dominated by the deeper subsurface layers owing to deeper penetration at these frequencies. They did not further probe for a satisfactory explanation for the reduction in planetary regolith temperature with depth. When emissivity of an object is close to unity at a particular wavelength, its $T_{\mathrm{b}}$ approaches to its physical temperature. In the case of Venus, the optical depth of its dense atmosphere at decimeter and meter wavelengths is much lower than unity and the observed emission is expected to be generated from its surface and subsurface with certain depth.

The reduction in $T_{\mathrm{b}}$ due to the atmosphere, ionosphere and the variation of dielectric values with decreasing frequencies are not expected to be significant. Radiative transfer model is an effective tool for computing thermal emission at microwave and radio wavelengths by accounting for the detailed variation of temperature and dielectric properties with the depth of the terrain as well as with altitude of the atmosphere of Venus. Further studies are needed to explain the lower values of $T_{\mathrm{b}}$ at frequencies $<1 \mathrm{GHz}$ (meter wavelengths), where emission arises predominantly from a region further down the surface owing to deeper penetration.

\section{CONCLUSION}

The first interferometric imaging observations of Venus at frequencies below $620 \mathrm{MHz}$ are presented here. These observations of thermal emission from Venus were conducted using the GMRT. The analyses of these data revealed that brightness temperature of Venus decreases with increasing wavelength as $526 \mathrm{~K} \pm 22,409 \mathrm{~K} \pm 33$, and $<426 \mathrm{~K}$ at $606,332.9$, and $239.9 \mathrm{MHz}$, respectively. These values are consistent with values of about $498 \mathrm{~K}$ and $523 \mathrm{~K}$ measured at 608 and $430 \mathrm{MHz}$, respectively by previous workers during the 1970s, but are much lower than those measured at higher frequencies, e.g., $679.9 \mathrm{~K} \pm 13.6$ at $4.86 \mathrm{GHz}$ using the VLA. The microwave observations ( $\mathrm{cm}$ wavelengths) of $T_{\mathrm{b}}$ of Venus has been explained earlier by considering emission from its atmosphere and surface. The observed variation of the $T_{\mathrm{b}}$ at low microwave frequencies $(<1 \mathrm{GHz})$ can only be 
explained with further radiative transfer studies, as in this frequency regime, the emission is dominated by the surface/subsurface of the planetary regolith.

\section{ACKNOWLEDGEMENT}

Authors thank Dr. Dharam Vir Lal, NCRA-TIFR, Dr. K Krishnamoorthy, former Director, SPL, VSSC and Dr. Nizy Mathew, SPL, VSSC for many valuable discussions. We thank the staff of the GMRT who made these observations possible. The GMRT is run by the National Centre for Radio Astrophysics of the Tata Institute of Fundamental Research. Finally, authors thank the anonymous referees for their constructive comments and valuable suggestions. Mr. Nithin Mohan is supported by ISRO Research Fellowship.

\section{REFERENCE}

Baars, J. W. M., Genzel, R., Pauliny-Toth, I. I. K., Witzel, A., 1977. The Absolute Spectrum of Cas A. An Accurate Flux Density Scale and a Set of Secondary Calibrators. Astron. Astrophys. 61 (99-106).

Basilevsky, A. T., Pronin, A. A., Ronca, B., Kryuchkov, V. P., Sukhanov, A. L., Markov, S., 1986. Styles of Tectonic Deformations on Venus: Analysis of Venera 15 and 16 Data. J. Geophys. Res. 91 (399-411).

Butler, B. J., Steffes, P. G., Suleiman, S. H., Koldoner, M. A., Jenkins, J. M., 2001. Accurate and Consistent Microwave Observations and their Implications. Icarus 154 (226-238).

Campbell, B. A., 1994. Merging Emissivity and SAR Data for Analysis of Venus Dielectric Properties. Icarus 112 (187-203).

Campbell, D. N., Head, J. W., Hine, A. A., Harmon, J. K., Senske, D. A., Fisher, P. C., 1989. Styles of Volcanism on Venus: New Arecibo High Resolution Radar Data. Science 246 (373-377).

Campbell, M. J., Ulrichs, J., 1969. Electrical Properties of Rocks and Their Significance for Lunar Radar Observations. J. Geophys. Res. 74 (5867-5881).

Carpenter, R. L., 1964. Study of Venus Surface by C.W. Radar. Astron. J. 69 (1-11).

Chengalur, J. N., 2013. NCRA Technical Report, NCRA/COM/OD, Tech. rep., National Centre for Radio Astrophysics, Pune 411007, India.

Condon, J. J., Jauncey, D. L., Yerbury, M. J., 1973. The Brightness Temperature of Venus at 70 Centimeters. Astrophys. J. 183 (1075-1080).

Fjeldbo, G., Kliore, A. J., Eshleman, V. R., 1971. The Neutral Atmosphere of Venus as Studied With the Mariner V Radio Occultation Experiment. Astron. J. 73 (123-140).

Florenskii, K. P., Bazilevsky, A. T., Kruchkyov, V. P., Kuzmin, O. V., Nikolaeva, Pronin, A A Chernaya, I. M., Selivanov, A. S., Naraeva, M. K., Tyuflin, Y. S., 1982. Analysis of the Panoramas of the Venera 13 and Venera 14 Landing Sites. Sov. Astron. Lett. 8 (233-234).

Ford, P. G., Pettengill, G. H., 1983. Venus: Global Surface Radio Emissivity. Science 220 (1379-1381).

Goldstein, R. M., Carpenter, R. L., 1963. Rotation of Venus: Period Estimated from Radar Measurements. Science 139 (910-911).

Haslam, C. G. T., Salter, C. J., Stoffel, H., Wilson, W. E., 1983. A 408 MHz All-Sky Continuum Survey II - The Atlas of Contour Maps. Astron. Astrophys. Suppl. Series 47 (1-142).

Herman, J., Hartle, R., Bauer, S., 1971. The Dayside Ionosphere of Venus. The Planet. Space Sci. 19.

James, J., Ingalls, R., 1967. Radar Observation of Venus at $38 \mathrm{Mc} / \mathrm{sec}$. The Astron. Journ. 72.

James, J., Ingalls, R., Rainville, L., 1967. Radar Echos from Venus at 38 $\mathrm{Mc} / \mathrm{sec}$. The Astron. Journ. 72.

Klemperer, W.K.and Ochs, G., Bowles, K., 1964. Radar Echos from Venus at $50 \mathrm{Mc} / \mathrm{sec}$. The Astron. Journ. 69.

Kuzmin, A., 1964. Radio Physical Investigations of Venus. In: Physics, All - Union Institute of Scientific and Technical Information. Academy of Science USSR, Moscow.

Kuzmin, A., 1967. Concerning a Model of Venus with Cold Absorbing Atmosphere. Izv. Vyssh. Ueheb. Zaved. Radiofiz. 7, 1021-1031.

Kuzmin, A. D., 1983. Radio Astronomical Studies of Venus. In: Venus. University of Arizona Press, Tucson, Arizona, pp. 37-44.
Markiewicz, W. J., Titov, D. V., Limaye, S. S., Keller, H. U., Ignatiev, N., Jaumann, R., Thomas, N., Michalik, H., Moissl, R., Russo, P., 2007. Morphology and Dynamics of the Upper Cloud Layer of Venus. Nature 450 (633-636).

Marov, M. Y., 1978. Results of Venus Missions. Annu. Rev. Astron. Astrophys. 16 (141-169).

Muhleman, D. O., Berge, G. L., Orton, G. S., 1973. The Brightness Temperature of Venus and the Absolute Flux-Density Scale at 608 MHz. Astrophys. J. 183 (1081-1085).

Muhleman, D. O., Orton, G. S., Berge, G. L., 1979. A Model of the Venus Atmosphere from Radio, Radar, and Occultation Observations. Astrophys. J. 234 (733-745).

Perley, R. A., Butler, B. J., 2013. An Accurate Flux Density Scale From 1 to $50 \mathrm{GHz}$. Astrophys. J. Supp. Series, 204:19(20 pp).

Pettengill, G. H., Eliason, E., Ford, P. G., Loriot, G. B., Masursky, H., McGill, G. E., 1980. Pioneer Venus Radar Results: Altimetry and Surface Properties. J. Geophys. Res. 85 (8261-8270).

Pettengill, G. H., Ford, P. G., Chapman, B. D., 1988. Venus: Surface Electromagnetic Properties. J. Geophys. Res. 93 (14,881-14,892).

Pettengill, G. H., Ford, P. G., Johnson, W. T. K., Raney, R. K., Soderblom, L. A., 1991. Magellan: Radar Performance and Data Products. Science 252 (260-265).

Pettengill, G. H., Ford, P. G., Wilt, R. J., 1992. Venus Rurface Radiothermal Emission as Observed by Magellan. J. Geophys. Res. 97 (13,09113,102).

Phillips, R. J., Malin, M. C., 1983. The interior of venus and tectonic implications. In: Venus. University of Arizona Press, Tucson, Arizona, pp. 159-214.

Prasad, J., Chengalur, J. N., 2012. FLAGCAL: a Flagging and Calibration Package for Radio Iterferometric Data. Exp. Astron. 33 (157171).

Reich, P., Reich, W., 1988. Spectral index variations of the galactic radio continuum emission - evidence for a galactic wind. Astron. Astrophys. 196, 211-226.

Scaife, A. M. M., Heald, G. H., 2012. A Broadband Flux Scale for Low Frequency Radio Telescopes. Mon. Not. R. Astron. Soc. 423 (30-34).

Seiff, A., Kirk, D. B., Young, R. E., Blanchard, R. C., Findlay, J. T., Kelley, G. M., Soreruer, S. C., 1980. Measurements of the Thermal Structure and Thermal Contrasts in the Atmosphere of Venus, and Related Dynamical Observations: Results from the four Pioneer Venus probes. J. Geophys. Res. 85 (7903-7933).

Sinclair, A. C. E., Basart, J. P., Buhl, D., Gale, W. A., Liwshitz, M., 1970. Preliminary Results of Interferometric Observations of Venus at $11.1 \mathrm{~cm}$ Wavelength. Radio Science 5 (347-354).

Surkov, Y. A., 1983. Studies of venus rocks by veneras 8,9 and 10. In: Venus. University of Arizona Press, Tucson, Arizona, pp. 154-158.

Swarup, G., Ananthkrishnan, S., Kapahi, V. K., Rao, A. P., Subrahmanya, C. R., Kulkarni, V. K., 1991. The Giant Metrewave Radio Telescope. Current Science 60 (90-105).

Taylor, G. B., Carilli, C. L., Perley, R. A., 1999. Synthesis Imaging in Radio Astronomy II. Vol. 180. Astronomical Society of the Pacific Conference Series.

Vinogradov, A. P., Florenskii, K. P., Bazilevskii, A. T., Selivanov, A. S., 1976. First Panoramic Pictures of Venus: Preliminary Image Analysis. Sov. Astron. Lett. 2 (67-71).

Warnock, W. W., Dickel, J. R., 1972. Venus: Measurements of Brightness Temperatures in the 7-15-cm Wavelength Range and Theoretical Radio and Radar Spectra for a two-layer Subsurface Model. Icarus 17 (682-691). 